\title{
Preenchimento de variáveis nas declarações de óbitos por causas externas de crianças e adolescentes no Recife, de 1979 a 1995
}

\author{
Analysis of child and adolescent mortality \\ reporting in Recife from 1979 to 1995
}

Maria Dilma de A. Barros 1

Ricardo Ximenes 2,3

Maria Luiza C. de Lima ${ }^{3}$

\footnotetext{
1 Departamento de Medicina Social, Faculdade de Ciências Médicas, Universidade Federal de Pernambuco. Rua Arnóbio Marques 310, Recife, $P E$

50100-130, Brasil.

dilma@elogica.com.br

2 Departamento de Medicina Tropical, Hospital das

Clínicas, Universidade Federal de Pernambuco. Av. Morais Rego s/no Cidade Universitária bloco A, Recife, PE 50670-620, Brasil. rximenes@elogica.com.br 3 Coordenação de Pós-Graduação, Faculdade de Ciências Médicas, Universidade Federal de Pernambuco. Rua Arnóbio Marques 310, Recife, $P E$

50100-130, Brasil.
}

\begin{abstract}
This article analyzes the completion of death certificates related to external causes in children and adolescents residing in Recife, Pernambuco State, Brazil, from 1979 to 1995. The analysis focused on the extent to which the following variables were filled in: personal data (occupation, level of schooling, medical care, confirmation of diagnosis by post-mortem, and type of violence) and place (location and municipality of both occurrence and death). Using the chisquared method, quantitative and/or qualitative flaws were found in most of the variables analyzed. In 1995, the "schooling" and "medical care" variables were recorded in only 5.7\% and 17.9\% of cases, respectively. Mismatches were observed between data on place of death and medical care, as well as between place of accident and number of accidents. The results suggest a dissociation between the objective of including the variable in the death certificate and its social function. The study provides the public sector with support for improved collecting and critical analysis of data in the mortality information system.
\end{abstract}

Key words Information Systems; Mortality Registries; Cause of Death

Resumo O artigo analisa o preenchimento de variáveis das declarações de óbitos (DO) por causas externas de crianças e adolescentes residentes no Recife, de 1970 a 1995. Analisou-se o grau de preenchimento das variáveis de pessoa (ocupação, grau de instrução, assistência médica, confirmação do diagnóstico por necropsia e tipo de violência) e de lugar (local e município de ocorrência do óbito e local do acidente) por intermédio do Qui-quadrado de tendência. Verificou-se deficiência na quantidade e ou qualidade no preenchimento para grande parte das variáveis analisadas. As variáveis grau de instrução e assistência médica alcançaram em 1995 percentuais de preenchimento de $5,7 \%$ e 17, 9\% respectivamente. Observou-se incompatibilidade entre os dados para local de ocorrência do óbito e assistência médica, bem como entre os de local do acidente e o número de acidentes. Os resultados encontrados sugerem dissociação entre o objetivo da inclusão da variável na DO e o seu uso social. O trabalho oferece subsídios ao setor público para melhoria da coleta e crítica dos dados do sistema de informação de mortalidade.

Palavras-chave Sistemas de Informação; Registros de Mortalidade; Causa da Morte 


\section{Introdução}

A apreensão da violência por meio da mortalidade tem como fonte de dados o Sistema de Informação de Mortalidade (SIM), que teve seu início com a implantação em nível nacional de um modelo padronizado de declaração de óbito em 1976. O atestado médico do óbito, parte integrante do mesmo, foi elaborado segundo o padrão internacionalmente recomendado pela Organização Mundial da Saúde (OMS) em 1948 e adotado pelo Brasil desde 1950 (MS, 1997).

Quando da informatização do sistema de mortalidade, o Ministério da Saúde (MS) definiu as variáveis que entrariam na declaração de óbito (DO), e essas foram classificadas para efeito de crítica e correção dos dados da seguinte forma: indispensáveis - aquelas cuja falta implica não-computação pelo sistema - ano do óbito e tipo de óbito; essenciais - as mais utilizadas em estudos de mortalidade e que têm prioridade para crítica e correção dos dados sexo, idade, município de ocorrência, município de residência, causa básica e tipo de violência; secundárias - aquelas que, apesar da grande importância pelo menor uso e pela maior dificuldade de se conseguirem níveis satisfatórios de qualidade, não têm prioridade para crítica e correção - todas as demais (MS, 1997).

Os dados de mortalidade por causas externas têm como fontes oficiais as Secretarias de Segurança Pública e as Secretarias Municipais e Estaduais de Saúde. Órgãos com atribuições, objetivos e funções sociais distintas, fortemente burocratizados e onde a tarefa de informar está deslocada de sua função social, não sendo, portanto, considerada importante (Njaine et al., 1997). Desta forma, já na geração dos dados há uma considerável perda resultante da não-definição das responsabilidades que cabem a cada uma dessas instituições. Também os diferentes entendimentos que têm sobre um mesmo ponto interferem na produção dos dados. Para os peritos do Instituto de Medicina Legal (IML), esclarecer as circunstâncias em que ocorreu a morte significa determinar a causa jurídica do óbito - podendo esta ser utilizada para fins judiciais -, enquanto para os Serviços de Saúde, esclarecer essas circunstâncias significa conhecer o início do processo que levou ao óbito, o ponto de intervenção por excelência (Njaine et al., 1997). Souza et al. (1996) discutiram a qualidade da informação sobre a mortalidade por causas externas desde sua geração até sua divulgação: sobre o primeiro ponto salientaram a deficiente formação dos profissionais que fazem os registros dos dados e a desconexão com o uso social da informa- ção; sobre o segundo, enfatizaram a insuficiência qualitativa do material divulgado, que obriga a utilização de artifícios técnicos para minimizar as deficiências.

Enfatiza o MS quando da apresentação das estatísticas de mortalidade, serem elas indispensáveis para a elaboração e análise dos principais indicadores de saúde. Acrescenta que os dados fornecidos quanto à idade, ao sexo, ao estado civil, à ocupação, à naturalidade e ao local de residência, se por um lado servem para identificar o falecido, constituem-se, por outro, em elementos preciosos para os estudos epidemiológicos (MS, 1997). Quanto a essas variáveis, que ajudariam a traçar o padrão de mortalidade, os estudos vêm referindo deficiência no seu preenchimento, inclusive em países desenvolvidos como os Estados Unidos (Comstock \& Markush, 1986; Hanzlick, 1997). No Brasil, estudos têm quantificado e discutido as implicações dos altos percentuais de ignorados encontrados para essas variáveis (Mello-Jorge, 1981; Souza, 1995b; Souza et al., 1996; Oliveira \& Pereira, 1997; Njaine et al., 1997; Souza et al., 1997). Para o Brasil e o Município do Rio de Janeiro em 1990, percentuais de ignorados acima de $50 \%$ foram referidos para a variável local do acidente. Para o Recife, em 1990, foram as variáveis instrução e assistência médica que tiveram percentuais semelhantes (Souza et al., 1996).

Sabe-se que uma elevada proporção de informação ignorada pode ser um indicador de qualidade dos dados, contribuindo para o desconhecimento da realidade das estatísticas de mortalidade (Souza et al., 1996; Njaine et al., 1997; Oliveira \& Pereira, 1997).

Segundo a Associação Brasileira de Saúde Coletiva (ABRASCO, 1997), existe o entendimento mas não a prática de que as informações públicas não pertencem aos órgãos responsáveis pelos sistemas, mas ao cidadão brasileiro. Todavia, a grande evolução técnica recente vem favorecendo o acesso aos bancos de dados e, na medida em que estes sofrem uma maior demanda, pode advir uma melhor análise e uma maior exigência de compatibilidade por parte dos usuários. Isto, por sua vez, poderá propiciar o repensar do Sistema de Informação em Saúde (SIS) em todas as suas etapas (ABRASCO, 1997).

No SIM em particular, a observação de suas inconsistências vem conduzindo a preocupação com outras variáveis não objeto de crítica pelo sistema como ocupação, local de ocorrência do evento, entre outros (ABRASCO, 1997). Vêm sendo também discutidas alterações no fluxo visando aumentar a agilidade, para que o óbito possa ser utilizado como instrumento de 
vigilância epidemiológica, como vem sendo feito pela Secretaria Municipal de Saúde de São Paulo, pelo Projeto de Melhoria de Informações de Mortalidade (ABRASCO, 1997).

Njaine et al. (1997) alertam para que a melhoria da qualidade da informação não seja vista apenas como uma questão técnica, mas sim como uma possibilidade de capacitar a sociedade a tomar decisões coerentes com os seus problemas.

A proposta neste estudo de avaliar-se o SIM, no tocante às causas externas, considerou os aspectos: escassez de trabalhos publicados para Recife avaliando o preenchimento de variáveis outras da DO que não causa básica, sobretudo seu comportamento no tempo e a possibilidade de trazer subsídios ao setor público para melhoria da coleta e crítica dos dados do SIM - favorecendo pois, a produção de dados mais fiéis que, divulgados, permitam à sociedade não só conhecer melhor o seu processo de violência como assumir as decisões mais adequadas para enfrentá-lo.

\section{Metodologia}

\section{Definição de variáveis}

Observou-se o comportamento do grau de preenchimento das variáveis relativas:

a) à pessoa (ocupação, grau de instrução, assistência médica, confirmação do diagnóstico por necropsia e tipo de violência);

b) a lugar (local e município de ocorrência do óbito e local do acidente).

\section{Coleta de dados}

Os dados relativos ao período de 1979 a 1992 foram obtidos a partir da transferência dos dados contidos em CD-ROM do MS, editado em 31/05/1996, com dados definitivos para Pernambuco até 1992 (MS, 1996). Para os anos de 1993 a 1995, foram utilizadas as informações constantes no banco de dados de mortalidade da Secretaria Estadual de Saúde de Pernambuco (SES-PE, 1997). Quando o MS editou novo CD-ROM com os dados definitivos para Pernambuco até 1995, as informações referentes aos anos de 1993, 1994 e 1995 foram comparadas com aquelas da SES/PE, não tendo sido encontradas diferenças, ou estas não foram importantes.

\section{Processamento de dados}

Para verificação do grau de preenchimento das variáveis das DO na série estudada, foram utilizados relatórios obtidos com o programa $T A B D O$, onde cada variável aparece com as mesmas categorias existentes nas DO. No MINI$T A B$ foram elaboradas planilhas com duas categorias: sim e não. O "sim" correspondendo ao preenchimento de qualquer das opções, exceto ignorado. Apesar de considerar-se que ignorado e sem informação têm significados diferentes, não há como separá-los nos relatórios. O "não" correspondeu à soma dos sem informação com os assinalados como ignorados nas variáveis das DO.

\section{Plano de descrição e análise dos dados}

Para verificar a variabilidade do grau de preenchimento das variáveis no tempo, foi usado o teste de $\chi^{2}$ de tendência, que permite identificar se o aumento ou decréscimo observado ocorreu de forma homogênea ao longo do tempo. Quando o teste de $\chi^{2}$ não se mostrou adequado pela presença de diversos valores menores que dois em linhas adjacentes (Armitage \& Berry, 1990), outro teste de verificação de tendência foi utilizado - o teste s, ou do sinal (Noether, 1983). Para todos os testes de significância estatística utilizados, foi tomado como ponto de corte o valor correspondente a $5 \%$, ou seja, os resultados foram considerados significantes quando o valor de $\mathrm{p}<0,05$.

\section{Resultados}

Analisou-se em termos quantitativos o preenchimento de variáveis selecionadas das DO e, dependendo dos resultados, discutiu-se e avaliou-se a questão da qualidade.

\section{Variáveis relativas à pessoa}

Os percentuais de preenchimento das variáveis relativas à pessoa encontram-se na Tabela 1. Quanto à instrução, que já apresentava uma porcentagem de preenchimento baixa (inferior a $40 \%$ ) no inicio da série, chegou, em 1995, a apresentar um percentual 6 vezes menor $\left(\chi^{2} \mathrm{de}\right.$ tendência $=78,61, \mathrm{p}<0,05)$. Ocupação apresentou um aumento dos valores informados de 1,7 vezes $\left(\chi^{2}\right.$ de tendência $\left.=152,38, \mathrm{p}<0,05\right)$. No entanto, vale ressaltar que $23,1 \%$ das DO não tinham ainda esse item completo em 1995. Quanto à assistência médica, caiu a menos de um quarto o seu percentual de preenchimento 
Tabela 1

Número e percentual de preenchimento de variáveis relativas à pessoa, nas declarações de óbitos por causas externas, em menores de 20 anos. Recife, Pernambuco, 1979-1995.

\begin{tabular}{|c|c|c|c|c|c|c|c|c|c|c|}
\hline & \multicolumn{2}{|c|}{ Instruçãa ${ }^{1}$} & \multicolumn{2}{|c|}{ Ocupação } & \multicolumn{2}{|c|}{$\begin{array}{l}\text { Assistência } \\
\text { médica } 1\end{array}$} & \multicolumn{2}{|c|}{ Necrópsia } & \multicolumn{2}{|c|}{$\begin{array}{l}\text { Tipo de } \\
\text { violência }\end{array}$} \\
\hline & $\mathrm{n}$ & $\%$ & $\mathrm{n}$ & $\%$ & $\mathrm{n}$ & $\%$ & $\mathrm{n}$ & $\%$ & $\mathrm{n}$ & $\%$ \\
\hline 1979 & 63 & 38,4 & 56 & 45,2 & 174 & 81,7 & 212 & 99,5 & 97 & 100,0 \\
\hline 1980 & 71 & 43,3 & 32 & 25,4 & 185 & 83,0 & 222 & 99,6 & 123 & 99,2 \\
\hline 1981 & 50 & 35,0 & 60 & 57,1 & 152 & 83,5 & 182 & 100,0 & 133 & 99,3 \\
\hline 1982 & 67 & 43,0 & 69 & 56,6 & 170 & 81,7 & 205 & 98,6 & 176 & 98,3 \\
\hline 1983 & 48 & 31,6 & 70 & 56,0 & 126 & 59,2 & 212 & 99,5 & 187 & 98,9 \\
\hline 1984 & 51 & 27,0 & 90 & 56,6 & 150 & 60,7 & 246 & 99,6 & 201 & 95,7 \\
\hline 1985 & 39 & 19,8 & 117 & 73,1 & 94 & 38,4 & 245 & 100,0 & 203 & 94,9 \\
\hline 1986 & 56 & 25,5 & 133 & 70,0 & 74 & 25,6 & 286 & 99,0 & 217 & 95,6 \\
\hline 1987 & 43 & 22,6 & 109 & 69,0 & 59 & 25,8 & 229 & 100,0 & 159 & 98,2 \\
\hline 1988 & 57 & 28,9 & 107 & 67,3 & 99 & 42,0 & 236 & 100,0 & 6 & 4,0 \\
\hline 1989 & 69 & 27,5 & 148 & 70,1 & 169 & 53,5 & 316 & 100,0 & 227 & 95,8 \\
\hline 1990 & 39 & 17,0 & 157 & 76,6 & 105 & 38,8 & 269 & 99,3 & 255 & 98,8 \\
\hline 1991 & 32 & 13,8 & 160 & 76,6 & 47 & 17,5 & 247 & 91,8 & 244 & 94,6 \\
\hline 1992 & 46 & 28,8 & 113 & 83,1 & 26 & 13,8 & 182 & 96,3 & 174 & 97,2 \\
\hline 1993 & 110 & 46,0 & 154 & 74,4 & 82 & 27,1 & 302 & 99,7 & 291 & 100,0 \\
\hline 1994 & 29 & 12,3 & 164 & 77,7 & 42 & 15,6 & 266 & 98,5 & 237 & 92,9 \\
\hline 1995 & 15 & 5,7 & 190 & 76,9 & 54 & 17,9 & 302 & 100,0 & 284 & 100,0 \\
\hline
\end{tabular}

Fontes: MS, 1996 (para os dados de 1979 a 1992); SES-PE, 1997 (para os dados de 1993 a 1995).

Para a variável Instrução, o percentual foi calculado em relação aos óbitos por causas externas de 5 - 19 anos.

Para a variável Ocupação, o percentual foi calculado em relação aos óbitos de 10 -19 anos por causas externas. Assistência Médica e Necropsia tiveram seus percentuais calculados em relação aos óbitos de 0 - 19 anos por causas externas. O percentual para Tipo de Violência foi calculado em relação aos óbitos de 0 - 19 anos com tipo de violência definido.

1 Como Ignorado para essas variáveis constitui-se em uma das opções de preenchimento, seu valor foi somado ao de Sem Informação.

em se considerando os anos extremos da série, chegando a apresentar valores inferiores a $20 \%$ $\left(\chi^{2}\right.$ de tendência $\left.=750,95, \mathrm{p}<0,05\right)$. O item das DO correspondente à confirmação do diagnóstico por necropsia apresentou-se completo em quase $100 \%$ durante toda série (teste do sinal = $0,247 \mathrm{p}>0,05)$. Comportamento similar apresentou a variável tipo de violência, exceto para o ano de 1988 , com apenas $4,0 \%$ das DO do estudo assinaladas (teste $\mathrm{s}=0,194, \mathrm{p}>0,05$ ).

\section{Variáveis relativas a lugar}

O comportamento do preenchimento das variáveis relativas a lugar pode ser observado na Tabela 2. Os percentuais encontrados para local de ocorrência do óbito foram elevados e variaram pouco ao longo da série. O teste do sinal mostrou que não se caracterizou tendência ( $p>0,05)$. Para altas percentagens de preenchimento da variável local de ocorrência do óbito, eram esperados também altos percentuais para o preenchimento da variável relativa à assistência médica, uma vez que se sabendo onde ocorreu o óbito - as opções na DO são hospital, via pública, domicílio e outro- seria muito provável que se obtivesse também a informação de ter ou não sido prestada assistência médica entre o evento e o óbito. O cruzamento dos dados dessas duas variáveis revelou entre os óbitos hospitalares percentuais de $2,1 \%$ a $11,9 \%$ sem assistência médica e de $1,4 \%$ a $81,4 \%$ com assistência médica ignorada. Quanto ao local do acidente, quando se consideraram os anos extremos da série, os percentuais encontrados em relação ao total de acidentes mostraram um decréscimo de quase metade ( $\chi^{2}$ de tendência $\left.=30,11, \mathrm{p}<0,05\right)$. Contudo, chamou atenção o fato de que os números absolutos encontrados para local do acidente nas causas externas como um todo se apresentavam superiores aos observados para os acidentes até o ano de 1990, após o que passaram a ser iguais. Evidenciando assim que para outros tipos de causas externas estava sendo preenchido também o item "local do acidente". Os percentuais de homicídios com local de acidente assinalado foram superiores a $30 \%$ entre 
Número e percentual de preenchimento de variáveis relativas a lugar, nas declarações de óbitos por causas externas, em menores de 20 anos. Recife, Pernambuco, 1979-1995.

\begin{tabular}{|c|c|c|c|c|c|c|c|c|}
\hline & \multicolumn{2}{|c|}{$\begin{array}{l}\text { Local de ocorrência } \\
\text { do óbito }\end{array}$} & \multicolumn{6}{|c|}{ Local do acidente 1} \\
\hline & \multirow[b]{2}{*}{$\mathrm{n}$} & \multirow[b]{2}{*}{$\%$} & \multicolumn{2}{|c|}{ Nas causas externas } & \multicolumn{2}{|c|}{ Nos acidentes } & \multicolumn{2}{|c|}{ Nos homicídios } \\
\hline & & & $\mathrm{n}$ & $\%$ & $n$ & $\%$ & $\mathrm{n}$ & $\%$ \\
\hline 1979 & 212 & 99,5 & 66 & 31,0 & 56 & 80,0 & 7 & 30,4 \\
\hline 1980 & 223 & 100,0 & 94 & 42,2 & 78 & 90,7 & 13 & 41,9 \\
\hline 1981 & 181 & 99,5 & 91 & 50,0 & 74 & 74,0 & 14 & 48,3 \\
\hline 1982 & 208 & 100,0 & 125 & 60,1 & 107 & 73,3 & 17 & 53,1 \\
\hline 1983 & 213 & 100,0 & 134 & 62,9 & 103 & 73,6 & 30 & 62,5 \\
\hline 1984 & 247 & 100,0 & 145 & 58,7 & 115 & 73,3 & 29 & 55,8 \\
\hline 1985 & 245 & 100,0 & 132 & 53,8 & 96 & 69,6 & 35 & 46,7 \\
\hline 1986 & 289 & 100,0 & 136 & 47,1 & 84 & 60,9 & 49 & 57,0 \\
\hline 1987 & 229 & 100,0 & 109 & 47,6 & 57 & 73,1 & 51 & 62,2 \\
\hline 1988 & 236 & 100,0 & 111 & 47,0 & 34 & 46,0 & 76 & 98,7 \\
\hline 1989 & 316 & 100,0 & 175 & 55,4 & 85 & 80,2 & 90 & 69,8 \\
\hline 1990 & 271 & 100,0 & 172 & 63,5 & 96 & 70,1 & 75 & 63,6 \\
\hline 1991 & 255 & 94,8 & 59 & 21,9 & 59 & 44,0 & - & - \\
\hline 1992 & 182 & 96,3 & 57 & 30,2 & 57 & 54,8 & - & - \\
\hline 1993 & 303 & 100,0 & 104 & 34,3 & 104 & 69,3 & - & - \\
\hline 1994 & 239 & 88,5 & 43 & 15,9 & 42 & 39,3 & - & - \\
\hline 1995 & 227 & 91,7 & 51 & 16,9 & 51 & 42,9 & - & - \\
\hline
\end{tabular}

Fontes: MS, 1996 (para os dados de 1979 a 1992); SES-PE, 1997 (para os dados de 1993 a 1995).

Para as variáveis Local de Ocorrência do Óbito e Local do Acidente nas causas externas, os percentuais

foram calculados em relação aos óbitos de 0 - 19 anos por causas externas. Para Local do Acidente

nos homicídios e nos acidentes, os percentuais foram calculados em relação aos óbitos de 0 - 19 anos pelo tipo especificado de causa externa para cada ano.

1 No caso de Local do Acidente, onde Ignorado constitui-se em uma das opções de preenchimento,

seus valores foram acrescidos aos de Sem Informação.

1979 e 1990, caindo a zero a partir de 1991. A tendência do grau de preenchimento foi crescente para o primeiro período referido $\left(\chi^{2} \mathrm{de}\right.$ tendência $=29,67, \mathrm{p}<0,05)$. Os suicídios foram também assinalados até 1990, mas, devido aos seus pequenos números, deixaram de ser apresentados. Chamou a atenção o fato de que para os óbitos classificados em outras violências nenhuma DO teve essa variável assinalada.

\section{Discussão}

De modo geral, os estudos realizados têm enfatizado as dificuldades no preenchimento da DO na sua parte IV, que diz respeito ao atestado médico do óbito, sobretudo no que se refere à causa básica de morte (Szwarcwald \& Castilho, 1986; Mello-Jorge, 1990; Laurenti \& Mello Jorge, 1993; Antunes et al., 1994; Souza, et al., 1996; Oliveira \& Pereira, 1997; Ladeira \& Guimarães, 1998). Outros têm analisado também o preenchimento de outras variáveis nos óbitos por causas externas (Mello-Jorge, 1980, 1981; Pereira \& Castro, 1981; Szwarcwald \& Castilho, 1986; Souza, 1995a; Souza et al., 1996; Njaine et al., 1997). Alguns desses autores alertaram, quando da apresentação dos dados, das implicações dos altos valores de ignorados encontrados para algumas delas (Souza, 1995b; Souza et al., 1996; Njaine et al., 1997). Os estudos para Recife, que incluíram a análise de outras variáveis além de causa básica, abordaram essencialmente idade, sexo e local de ocorrência do óbito (Lessa et al., 1995; Lima, 1995).

Nos Estados Unidos, como ocorre no Brasil, os autores detêm-se sobretudo na questão da causa básica (Kircher \& Anderson, 1987; Moyer et al., 1989; Messite et al., 1996), mas foram encontrados, na literatura revisada, estudos tratando do grau de preenchimento de outras variáveis e sua importância para ajudar a traçar o padrão de mortalidade (Comstock \& Markush, 1986; Hanzlick, 1997). 


\section{Variáveis relativas à pessoa}

A variável ocupação apresentou crescimento na quantidade do seu preenchimento ao longo da série estudada, enquanto que com instrução ocorreu o inverso. Souza et al. (1996) apresentaram a proporção de ignorados para ocupação e instrução para Brasil, Rio de Janeiro e Recife em 1990, nos óbitos por causas externas para a população geral. Para ocupação, os percentuais de ignorado para os três locais citados ficaram entre $20 \%$ e $25 \%$. Para instrução, esses valores foram superiores a $30 \%$ para Brasil e Rio de Janeiro, e bem mais altos para Recife $-86,5 \%$. Estes resultados referenciados para Recife foram concordantes com os encontrados neste trabalho. Oliveira \& Pereira (1997), discutindo as estatísticas de mortalidade em geral para o país, afirmaram não haver grandes problemas quanto ao preenchimento de algumas variáveis, entre elas ocupação e instrução. Os resultados deste estudo conduziram a conclusões divergentes da citada por esses autores.

Os estudos de mortalidade por causas externas têm, com freqüência, utilizado instrução como um dos fatores importantes na caracterização da população atingida (Mello-Jorge, 1988; Minayo \& Souza, 1993; Lima, 1995; Souza, 1995a). Por sua vez, Szwarcwald \& Castilho (1986), para as regiões administrativas do município do Rio de Janeiro em 1980, encontraram correlação positiva entre a taxa de homicídios e a proporção de analfabetos. Porém, diante de um percentual de mais de $90 \%$ de ignorados como o observado neste estudo para Recife em 1995, como caracterizar uma população conforme o grau de instrução?

A proporção de informação ignorada, quando elevada, contribui para o desconhecimento da realidade das estatísticas de mortalidade e pode ser considerada como um indicador de qualidade dos dados (Souza et al., 1996; Oliveira \& Pereira, 1997).

O item relativo ao diagnóstico ter sido confirmado por necropsia foi completado em mais de $90 \%$ neste trabalho. Situação melhor que a encontrada para os óbitos por causas externas no país em 1990 (78\%) (Souza et al., 1996) e também melhor que a do Rio de Janeiro para os óbitos de 0-19 anos, por essas mesmas causas, em 1993 (68\%) (Njaine et al., 1997).

Quanto à variável assistência médica, a análise dos dados sugere que não parece ter havido interesse em obtê-la. Os achados de mais de $60 \%$ sem informação, em mais da metade dos anos que compõem a série corroboram essa idéia. Oliveira \& Pereira (1997) citam assistência médica como uma das variáveis que com maior freqüência apresentam erros de preenchimento.

O baixo percentual de preenchimento encontrado em 1988 para a variável tipo de violência deve-se provavelmente a erro de digitação, uma vez que esse é o único valor aberrante na série e, sobretudo, porque se de fato estivessem esses óbitos assinalados como de tipo de violência ignorado na DO, a causa básica também seria ignorada e não uma das que compõem os grupos específicos de causas externas (acidentes, suicídios e homicídios). Sabe-se que isso não ocorreu porque ao observar-se na mesma série temporal do estudo o comportamento da causa básica desses óbitos, o ano de 1988 não apresenta comportamento atípico.

\section{Variáveis relativas a lugar}

Local de ocorrência do óbito foi uma variável que apresentou alto percentual de preenchimento ao longo de toda a série, semelhante a outros estudos realizados, seja direcionado a avaliar a quantidade de informação existente nas DO (Souza et al., 1996; Njaine et al., 1997) ou a caracterização da população atingida pela mortalidade por causas externas (Mello-Jorge, 1980, 1981; Lessa et al., 1995; Lima, 1995; Souza, 1995b; Souza et al., 1997).

Considerando que nas causas externas os óbitos não hospitalares ocorrem em sua maior parte na via pública, e, em parte pelas circunstâncias que os envolvem e em parte pela infraestrutura dos serviços de saúde não adequada a esse tipo de atendimento pode-se assumir esses óbitos como sem assistência médica entre o evento e o óbito. Por outro lado, para os óbitos hospitalares a guia de encaminhamento do corpo para o IML provavelmente fornece informação sobre ter sido ou não prestada assistência médica. Assim, como já foi dito anteriormente, para altos percentuais de preenchimento da variável local de ocorrência do óbito eram esperados também altos percentuais para a variável assistência médica. Os baixos percentuais de ignorados para local de ocorrência do óbito quando associados aos elevados para assistência médica sugerem inconsistência dos dados, sobretudo pelos valores encontrados para óbitos hospitalares sem assistência médica ou com assistência médica ignorada. Essa incompatibilidade dos dados mostra que na geração da informação na DO, pelo menos quanto à variável assistência médica, não há preocupação com o objetivo da sua inclusão. Ou seja, não há conexão entre a produção da informação e o seu uso social (Branco, 1995; Souza et al., 1996). 
No caso da variável local do acidente observou-se que havia mais casos com local do acidente preenchido que o número de acidentes, o que pode ser melhor verificado quando se quantificou o preenchimento de local do acidente para os outros tipos específicos de causas externas. Sem dúvida, é importante saber o local de ocorrência de todos os eventos violentos, tanto é que a CID-10 (OMS, 1993) tem para alguns códigos o seu quarto dígito direcionado a esse conhecimento. Porém, a análise desses números como se fossem todos correspondentes a acidentes constitui-se em uma distorção das estatísticas nos estudos de mortalidade. Por exemplo, poderia corresponder a homicídio ou suicídio parte dos valores ditos como de acidentes ocorridos em domicílio. A análise desses dados sugeriu que para os profissionais do IML essa variável devia ser preenchida para todos os tipos identificados de violência. Esse comportamento, que parece ter sido sistemático, reflete falta de conhecimento por parte de quem preencheu as DO e falta de crítica dos dados por parte dos serviços de saúde. A partir de 1991, embora hajam aumentado os percentuais de sem informação para local do acidente nos óbitos por esse tipo de causa, deixou de aparecer em DO por outras categorias de causas externas a variável local do acidente assinalada. A observação desses fatos parece indicar uma ação crítica por meio do programa de computação, que aceitaria a inclusão dessa variável apenas para os óbitos classificados como acidentais. Considerando essa hipótese verdadeira, não se pode negar uma melhoria na qualidade dos dados divulgados, embora não do preenchimento dos instrumentos que os geraram. Enfim, como assinalaram Souza et al. (1996), as dificuldades existem desde a geração até a divulgação de informações. Neste estudo foram analisadas algumas questões referentes à geração, refletindo a precária formação dos profissionais que lidam com os registros e a desconexão dessa tarefa com o uso social da informação.

\section{Agradecimento}

Agradecemos ao professor José Natal Figueiroa pela colaboração na análise estatística.
Em síntese, as variáveis que iniciaram a série com altos percentuais de preenchimento permaneceram como tal, as demais, com exceção para ocupação, pioraram o seu preenchimento. Após análise dos dados os autores consideram como uma possível explicação para o comportamento do preenchimento das variáveis das DO o entendimento que os profissionais do IML têm da sua responsabilidade na produção desses dados. Assim, algumas variáveis seriam entendidas como de sua responsabilidade direta, talvez por serem passíveis de determinação à luz da necropsia, e seriam preenchidas de modo sistemático - se foi realizada necropsia e tipo de violência por exemplos. Outras que preenchem de forma irregular entenderiam como de responsabilidade indireta porque necessitam retirar as informações do documento que acompanha o cadáver - guia de encaminhamento de hospital ou delegacia de polícia. Local de ocorrência do óbito, apesar de estar nesse segundo grupo de variáveis, teria um alto percentual de preenchimento por identificar muitas vezes o lugar que encaminhou o corpo ao IML.

A deficiência na quantidade ou qualidade do preenchimento para grande parte das variáveis analisadas aliada ao reconhecimento inclusive por parte do MS da importância das mesmas (MS, 1997) - para caracterização da população atingida pela mortalidade - permite algumas recomendações:

a) Que se promova, em curto prazo, uma discussão entre técnicos da SMS e do IML visando à melhoria do preenchimento de algumas variáveis das DO, tais como local e município de ocorrência do óbito e assistência médica.

b) Que seja realizada a crítica das variáveis outras que não causa básica por parte da Secretaria Municipal de Saúde (SMS) com correção, dentro das suas possibilidades, das distorções existentes.

c) Que a definição das variáveis para compor a DO contemple seu uso enquanto informação.

\section{Referências}

ABRASCO (Associação Brasileira de Saúde Coletiva), 1997. Compatibilização de bases de dados nacionais. Relatório da oficina de trabalho organizada pelo Grupo Técnico de Informação em Saúde e População da ABRASCO, São Paulo, 1997. Informe Epidemiológico do SUS, 3:25-33.

ANTUNES, M. B. C.; GENTILE, A. R.; SOARES, J. R. R. \& GUIMARÃES, M. J. B., 1994. Causas externas, uma realidade mal definida: Análise de uma rotina em 
serviço no resgate das informações. In: Congresso Brasileiro de Saúde Coletiva, Resumos, p. 7. Recife: ABRASCO.

ARMITAGE, B. \& BERRY, G., 1990. Statistical inference. In: Statistical Methods in Medical Research (P. Armitage \& G. Berry, eds.), pp. 93-140, Oxford: Blackwell Scientific Publication.

BRANCO, M. A. F., 1995. Sistema de Informação em Saúde em Âmbito Local e Organizações de Interesses Sociais: Um Estudo de Caso no Município do Rio de Janeiro. Dissertação de Mestrado, Rio de Janeiro: Instituto de Medicina Social, Universidade do Estado do Rio de Janeiro.

COMSTOCK, G. W. \& MARKUSH, R. E., 1986. Further comments on problems in death certification. American Journal of Epidemiology, 124:180-181.

HANZLICK, R., 1997. Death registration: History, methods and legal issues. Journal of Forensic Sciences, 2:265-269.

KIRCHER, T. \& ANDERSON, R. E., 1987. Cause of the death - Proper completion of the death certificate. JAMA, 258:349-352.

LADEIRA, R. M. \& GUIMARÃES, M. D., 1998. Análise da concordância da codificação de causa básica de óbito por acidentes de trânsito. Revista de Saúde Pública, 32:133-137.

LAURENTI, R. \& MELLO-JORGE, M. H. P., 1993. O Atestado de Óbito. São Paulo: Centro Brasileiro da Organização Mundial de Saúde para Classificações de Doenças em Português.

LESSA, F.; GUIMARÃES, M. J. B.; SANTOS, S.; PARENTE, S.; ANTUNES, A. \& AVELAR, I., 1995. Um retrato da violência em Recife em 1994. In: Congresso Brasileiro de Ciências Sociais em Saúde, Resumos, p. 133. Curitiba: ABRASCO.

LIMA, M. L. C., 1995. Violência e Morte: Diferenciais da Mortalidade por Causas Externas no Espaço Urbano de Recife, 1991. Dissertação de Mestrado, Salvador: Instituto de Saúde Coletiva, Universidade Federal da Bahia.

MELLO-JORGE, M. H. P., 1980. Mortalidade por causas violentas no Município de São Paulo, Brasil: II Mortes acidentais. Revista de Saúde Pública, 14: 475-508.

MELLO-JORGE, M. H. P., 1981. Mortalidade por causas violentas no Município de São Paulo, Brasil: III - Mortes intencionais. Revista de Saúde Pública, 15:165-193.

MELLO-JORGE, M. H. P., 1988. Investigação Sobre Mortalidade por Acidentes e Violências na Infância. Tese de Livre Docência, São Paulo: Faculdade de Saúde Pública, Universidade de São Paulo.

MELLO-JORGE, M. H. P., 1990. Situação atual das estatísticas oficiais relativas à mortalidade por causas externas. Revista de Saúde Pública, 24:217-223.

MESSITE, J. \& STELLMAN, S. D., 1996. Accuracy of death certificate completion: The need for formalized physician training. JAMA, 275:794-796.

MINAYO, M. C. S. \& SOUZA, E. R., 1993. Violência para todos. Cadernos de Saúde Pública, 13:405-414.

MOYER, I. A.; BOYLE, C. A. \& POLLOCK, D. A., 1989. Validity of death certification for injury - Related causes of death. American Journal of Epidemiology, 130:1024-1032.

MS (Ministério da Saúde), 1996. Sistema de Informação sobre Mortalidade, 1979-1992. Brasília: De- partamento de Informática do Sistema Único de Saúde.

MS (Ministério da Saúde), 1997. Mortalidade Brasil 1994. Brasília: Coordenação de Informações e Análise da Situação de Saúde, Centro Nacional de Epidemiologia, Fundação Nacional de Saúde.

NJAINE, K.; SOUZA, E. R.; MINAYO, M. S. C. \& ASSIS, S. G., 1997. A produção da (des)informação sobre violência: Análise de uma prática discriminatória. Cadernos de Saúde Pública, 13:405-414.

NOETHER, G. E., 1983. Casualidade, independência e correlação de postos. In: Introdução à Estatística: Uma Abordagem não Paramétrica (G. E. Noether, ed.), pp. 168-171, Rio de Janeiro: Guanabara Dois.

OLIVEIRA, H. \& PEREIRA, I. P. A., 1997. Estatística de mortalidade e nascidos vivos: Considerações sobre principais problemas. Informe Epidemiológico do SUS, 3:15-19.

OMS (Organização Mundial da Saúde), 1993. Manual da Classificação Estatística Internacional de Doenças e Problemas Relacionados à Saúde. 10a Revisão. São Paulo: Centro Colaborador da Organização Mundial da Saúde para Classificação de Doenças em Português/Edusp.

PEREIRA, M. G. \& CASTRO, E. S., 1981. Avaliação do preenchimento de declarações de óbitos: Brasília, D.F. (Brasil), 1977-1978. Revista de Saúde Pública, 5:14-19.

SES-PE (Secretaria de Saúde do Estado de Pernambuco), 1997. Dados sobre Mortalidade por Causas Externa no Município do Recife, 1993-1995. Recife: Divisão de Mortalidade, SES-PE.

SOUZA, E. R., 1995a. Homicídios: Metáfora de uma Nação Autofágica. Tese de Doutorado, Rio de Janeiro: Escola Nacional de Saúde Pública, Fundação Oswaldo Cruz

SOUZA, E. R., 1995b. Enfrentar a Violência um Desafio para o Estado. Recife: Secretaria de Saúde do Estado de Pernambuco. (mimeo.)

SOUZA, E. R.; ASSIS, S. G. \& SILVA, C. M. F. P., 1997. Violência no município do Rio de Janeiro: Áreas de risco e tendências da mortalidade entre adolescentes de 10 a 19 anos. Revista Panamericana de Salud Pública, 1:389-395.

SOUZA, E. R.; NJAINE, K. \& MINAYO, M. C. S., 1996. Qualidade da informação sobre violência: Um caminho para a construção da cidadania. Cadernos do Programa de Pós-graduação em Ciência da Informação, 2:104-112.

SZWARCWALD, C. L. \& CASTILHO, E. A., 1986. Mortalidade por causas externas no estado do Rio de Janeiro no período de 1976 a 1980. Cadernos de Saúde Pública, 2:19-41. 